ESAIM: COCV 20 (2014) 339-361

DOI: $10.1051 / \mathrm{cocv} / 2013066$
ESAIM: Control, Optimisation and Calculus of Variations

www.esaim-cocv.org

\title{
EXACT BOUNDARY SYNCHRONIZATION FOR A COUPLED SYSTEM OF 1-D WAVE EQUATIONS
}

\author{
Tatsien Li (Daqian Li) ${ }^{1}$, Bopeng RaO ${ }^{2}$ And Long Hu ${ }^{1}$
}

\begin{abstract}
Several kinds of exact synchronizations and the generalized exact synchronization are introduced for a coupled system of $1-\mathrm{D}$ wave equations with various boundary conditions and we show that these synchronizations can be realized by means of some boundary controls.
\end{abstract}

Mathematics Subject Classification. 35B37, 93B05, 93B07.

Received September 29, 2012. Revised May 24, 2013.

Published online February 6, 2014.

\section{INTRODUCTION}

Synchronization is a widespread natural phenomenon. Thousands of fireflies may twinkle at the same time; audiences in the theater can applaud with a rhythmic beat; pacemaker cells of the heart function simultaneously; and field crickets give out a unanimous cry-all these are phenomena of synchronization $[17,19]$.

In principle, synchronization happens when different individuals possess likeness in nature, that is, they conform essentially to the same governing equation, and meanwhile, the individuals should bear a certain coupled relation.

The phenomenon of synchronization was first observed by Huygens in 1665 [7]. The theoretical research on synchronization phenomena dates back to Fujisaka and Yamada's study of synchronization for coupled equations in 1983 [4]. The previous studies focused on systems described by ODEs, such as

$$
\frac{\mathrm{d} X_{i}}{\mathrm{~d} t}=f\left(X_{i}, t\right)+\sum_{j=1}^{N} A_{i j} X_{j} \quad(i=1, \ldots, N),
$$

where $X_{i}(i=1, \ldots, N)$ are $n$-dimensional vectors, $A_{i j}(i, j=1, \ldots, N)$ are $n \times n$ matrices, and $f(X, t)$ is an n-dimensional vector function independent of $i$. The right-hand side of $(1.1)$ shows that every $X_{i}(i=1, \ldots, N)$ possesses two basic features: satisfying a fundamental governing equation and bearing a coupled relation among one another.

\footnotetext{
Keywords and phrases. Exact null controllability, exact synchronization, exact synchronization by groups, exact null controllability and synchronization by groups, generalized exact synchronization.

1 School of Mathematical Sciences, Fudan University, Shanghai Key Laboratory for Contemporary Applied Mathematics; Nonlinear Mathematical Modeling and Methods Laboratory, Shanghai 200433, China.

dqli@fudan.edu.cn; 10110180015@fudan.edu.cn

2 Institut de Recherche Mathématique Avancée, Université de Strasbourg, 67084 Strasbourg, France. rao@math.u-strasbg.fr
} 
Our goal is to synchronize a hyperbolic system through boundary controls. Different from the ODE situation, the coupling of systems can then be fulfilled by coupling of the equations or (and) the boundary conditions, which has richer research implications, moreover, boundary controls can be used to realize our goal in a finite time.

Roughly speaking, we want to find a $T>0$ and some boundary controls on $[0, T]$, such that from the time $t=T$ on, the system states tend to the same. That is to say, we hope to achieve the synchronization of the system states not only at the moment $t=T$ under the action of boundary controls on $[0, T]$, but also when $t \geq T$ and withdrawing all the controls. This is forever, instead of short-lived, synchronization, as is desired in many actual applications.

Obviously, if the system has the exact boundary null controllability [11,14-16], it must have the exact boundary synchronization, but this is a trivial situation that should be excluded beforehand. The exact boundary null controllability here also means that the system states remain null not only at the moment $t=T$ under the action of boundary controls on $[0, T]$, but also when $t \geq T$ and withdrawing all the controls.

In this paper, we consider the exact boundary synchronization for a coupled system of 1-D wave equations with various boundary condition in the framework of classical solutions. The same problem for a coupled system of $n$-dimensional wave equations with Dirichlet boundary controls in the framework of weak solutions can be found in [13]. We point out that in 1-D case, thanks to the theory on semi-global classical solutions(see $[8,10]$ ), there is a unified theory on the exact boundary (null) controllability for a coupled system of wave equations with various kinds of coupled boundary conditions (see $[6,10,11,18]$ ), which enables us to get a complete result on the exact boundary synchronization. However, in the framework of weak solutions in n-dimensional case, for getting the corresponding exact boundary synchronization, we have to study the exact boundary (null) controllability for a coupled system of wave equations with every prescribed kind of boundary conditions separately, and, up to now, only the Dirichlet type of boundary conditions was discussed in [13]. In addition, the generalized exact boundary synchronization is introduced and discussed in this paper.

Precisely speaking, we will consider the following coupled system of wave equations

$$
\frac{\partial^{2} U}{\partial t^{2}}-\frac{\partial^{2} U}{\partial x^{2}}+A U=0
$$

where

$$
U=\left(u_{1}, \ldots, u_{N}\right)^{T}
$$

is an unknown vector function of $(t, x), A=\left(a_{i j}\right)$ is a $N \times N$ coupling matrix with constant elements. (1.2) can be written as

$$
\frac{\partial^{2} u_{i}}{\partial t^{2}}-\frac{\partial^{2} u_{i}}{\partial x^{2}}+\sum_{j=1}^{N} a_{i j} u_{j}=0 \quad(i=1, \ldots, N) .
$$

At the end $x=0$, we prescribe anyone of the following boundary conditions:

$$
\begin{aligned}
& x=0: \quad U=H(t) \quad \text { (Dirichlet type) } \\
& x=0: U_{x}=H(t) \quad \text { (Neumann type) } \\
& x=0: \quad U_{x}-B U=H(t) \quad \text { (Coupled third type) }
\end{aligned}
$$

where $B=\left(b_{i j}\right)$ is an $N \times N$ coupling matrix with constant elements, and

$$
H(t)=\left(h_{1}(t), \ldots, h_{N}(t)\right)^{T},
$$

the components of which will be totally or partially taken as boundary controls. 
Similarly, at the end $x=L$, since no boundary controls are concerned for one-sided control, we prescribe anyone of the following homogeneous boundary conditions:

$$
\begin{aligned}
& x=L: U=0, \\
& x=L: U_{x}=0, \\
& x=L: U_{x}+\bar{B} U=0,
\end{aligned}
$$

where $\bar{B}=\left(\bar{b}_{i j}\right)$ is an $N \times N$ coupling matrix with constant elements.

The initial condition is given by

$$
t=0: U=\Phi(x), \quad U_{t}=\Psi(x),
$$

where $\Phi(x)=\left(\varphi_{1}(x), \ldots, \varphi_{N}(x)\right)^{T}$ and $\Psi(x)=\left(\psi_{1}(x), \ldots, \psi_{N}(x)\right)^{T}$.

For the forward mixed initial-boundary value problem (1.2), (1.5), (1.7) and (1.8) of the coupled system of wave equations, by means of its reduction to the mixed initial-boundary value problem of a corresponding first order hyperbolic system and using the theory of global $C^{1}$ solutions to first order linear hyperbolic systems, we have (see $[6,10])$

Lemma 1.1. For any given $T>0$, assume that $\Phi \in\left(C^{2}[0, L]\right)^{N}, \Psi \in\left(C^{1}[0, L]\right)^{N}$ and $H \in\left(C^{2}[0, T]\right)^{N}$ (case $(1.5 \mathrm{a}))$ or $\left(C^{1}[0, T]\right)^{N}$ (cases $\left.(1.5 \mathrm{~b})-(1.5 \mathrm{c})\right)$ satisfy the conditions of $C^{2}$ compatibility at the points $(t, x)=$ $(0,0)$ and $(0, L)$, respectively (see Rem. 1.3). For any given coupling matrices $A, B$ and $\bar{B}$, the forward mixed initial-boundary value problem (1.2), (1.5), (1.7) and (1.8) admits a unique $C^{2}$ solution $U=U(t, x)$ on the domain $R(T)=\{(t, x) \mid 0 \leq t \leq T, 0 \leq x \leq L\}$.

Remark 1.2. In what follows, (1.5) always means anyone of (1.5a), (1.5b) and (1.5c), and it is similar for (1.7).

Remark 1.3. The conditions of $C^{2}$ compatibility at the point $(t, x)=(0, L)$ can be precisely written as

$$
\begin{aligned}
& \left\{\begin{array}{l}
\Phi(L)=0, \\
\Psi(L)=0, \\
\Phi^{\prime \prime}(L)=0,
\end{array} \text { for }(1.7 \mathrm{a}) ;\right. \\
& \left\{\begin{array}{l}
\Phi^{\prime}(L)=0, \quad \text { for }(1.7 \mathrm{~b}) ; \\
\Psi^{\prime}(L)=0,
\end{array}\right.
\end{aligned}
$$

or

$$
\left\{\begin{array}{l}
\Phi^{\prime}(L)+\bar{B} \Phi(L)=0, \\
\Psi^{\prime}(L)+\bar{B} \Psi(L)=0,
\end{array} \text { for }(1.7 \mathrm{c})\right.
$$

The conditions of $C^{2}$ compatibility at the point $(t, x)=(0,0)$ can be similarly obtained.

Remark 1.4. By Lemma 1.1, in order to guarantee the well-posedness of the forward mixed problem (1.2), (1.5), (1.7) and (1.8), no assumptions on the coupling matrices $A, B$ and $\bar{B}$ are needed.

Remark 1.5. For the backward mixed initial-boundary value problem (1.2), (1.5), (1.7) with the final condition

$$
t=T: U=\bar{\Phi}(x), \quad U_{t}=\bar{\Psi}(x) .
$$

where $\bar{\Phi}(x)=\left(\bar{\varphi}_{1}(x), \ldots, \bar{\varphi}_{N}(x)\right)^{T}$ and $\bar{\Psi}(x)=\left(\bar{\psi}_{1}(x), \ldots, \bar{\psi}_{N}(x)\right)^{T}$, the conclusion of Lemma 1.1 is still valid.

By Lemma 1.1 and using a constructive method, we have the following result on the exact boundary null controllability (also see $[6,18,20]$ ). 
Lemma 1.6. Let

$$
T>2 L \text {. }
$$

For any given initial state $(\Phi, \Psi) \in\left(C^{2}[0, L]\right)^{N} \times\left(C^{1}[0, L]\right)^{N}$, satisfying the conditions of $C^{2}$ compatibility at the point $(t, x)=(0, L)$, there exist boundary controls $H \in\left(C^{2}[0, T]\right)^{N}$ (case $\left.(1.5 \mathrm{a})\right)$ or $\left(C^{1}[0, T]\right)^{N}($ cases $(1.5 \mathrm{~b})-$ $(1.5 \mathrm{c})$ ) with support on $[0, T]$ at the end $x=0$, such that the corresponding mixed initial-boundary value problem (1.2), (1.5), (1.7) and (1.8) admits a unique $C^{2}$ solution $U=U(t, x)$ on $t \geq 0$, and as $t \geq T$ we have

$$
U(t, x) \equiv 0, \quad 0 \leq x \leq L
$$

In what follows, we will use Lemma 1.1 and Lemma 1.6 to consider the exact boundary synchronization, the exact boundary synchronization by groups, and the exact boundary null controllability and synchronization by groups in Section 2, Section 3 and Section 4, respectively, and the generalized exact boundary synchronization in Section 5, for the coupled system of wave equations (1.2), (1.5) and (1.7). Moreover, several remarks are given in Section 6.

\section{EXACT BOUNDARY SYNCHRONIZATION}

Definition 2.1. If there exists $T>0$ such that for any given initial state $(\Phi(x), \Psi(x))$, we can find some boundary controls with support on $[0, T]$ in $H(t)$, such that the corresponding mixed initial-boundary value problem (1.2), (1.5), (1.7) and (1.8) admits a unique $C^{2}$ solution $U=U(t, x)=\left(u_{1}(t, x), \ldots, u_{N}(t, x)\right)^{T}$ on $t \geq 0$, and as $t \geq T$ we have

$$
u_{1}(t, x) \equiv \ldots \equiv u_{N}(t, x) \stackrel{\text { def. }}{=} \widetilde{\widetilde{u}}(t, x), \quad 0 \leq x \leq L
$$

then the coupled system (1.2), (1.5) and (1.7) is said to possess the exact boundary synchronization or is said to be exactly synchronizable, and $\widetilde{\widetilde{u}}=\widetilde{\widetilde{u}}(t, x)$ is called the corresponding synchronizable state.

Obviously, if the system possesses the exact boundary null controllability, then it must be exactly synchronizable. However, this trivial situation should be excluded in the discussion.

Lemma 2.2. If the coupled system of wave equations (1.2), (1.5) and (1.7) is exactly synchronizable, but not exactly null controllable, then the coupling matrices $A, B$ and $\bar{B}$ should satisfy the following necessary conditions: The values of the sum of every row of these matrices, $\sum_{j=1}^{N} a_{i j}, \sum_{j=1}^{N} b_{i j}$ and $\sum_{j=1}^{N} \bar{b}_{i j}$ are independent of $i=1, \ldots, N$, namely,

$$
\begin{aligned}
& \sum_{j=1}^{N} a_{i j} \stackrel{\text { def. }}{=} \widetilde{\widetilde{a}} \quad(i=1, \ldots, N), \\
& \sum_{j=1}^{N} b_{i j} \stackrel{\text { def. }}{=} \widetilde{\widetilde{b}} \quad(i=1, \ldots, N), \\
& \sum_{j=1}^{N} \bar{b}_{i j} \stackrel{\text { def. }}{=} \widetilde{\widetilde{b}} \quad(i=1, \ldots, N),
\end{aligned}
$$

where $\widetilde{\widetilde{a}}, \widetilde{\widetilde{b}}$ and $\widetilde{\bar{b}}$ are constants independent of $i=1, \ldots, N$. 
Proof. By synchronization, there exists $T>0$ and a synchronizable state $\widetilde{\widetilde{u}}=\widetilde{\widetilde{u}}(t, x)$ such that $(2.1)$ holds. Then, it follows from (1.4) that for $t \geq T$ we have

$$
\frac{\partial^{2} \widetilde{\widetilde{u}}}{\partial t^{2}}-\frac{\partial^{2} \widetilde{\widetilde{u}}}{\partial x^{2}}+\left(\sum_{j=1}^{N} a_{i j}\right) \widetilde{\widetilde{u}}=0, \quad 0 \leq x \leq L, i=1, \ldots, N .
$$

In particular, for $t \geq T$ we get

$$
\left(\sum_{j=1}^{N} a_{i j}\right) \widetilde{\widetilde{u}}=\left(\sum_{j=1}^{N} a_{k j}\right) \widetilde{\widetilde{u}}, \quad 0 \leq x \leq L, i, k=1, \ldots, N .
$$

By the non exact null controllability, there exists at least an initial data $(\Phi, \Psi)$ for which the corresponding solution $U$, or equivalently $\widetilde{\widetilde{u}}$, does not identically vanish for $t \geq T$ whatever boundary controls $H$ are chosen. This leads to $(2.2)$. In the meantime, noting $(2.5)$, the synchronizable state $\widetilde{\widetilde{u}}=\widetilde{\widetilde{u}}(t, x)$ satisfies the following wave equation

$$
\frac{\partial^{2} \widetilde{\widetilde{u}}}{\partial t^{2}}-\frac{\partial^{2} \widetilde{\widetilde{u}}}{\partial x^{2}}+\widetilde{\widetilde{a} \widetilde{u}}=0
$$

where $\widetilde{\widetilde{a}}$ is given by $(2.2)$.

We now prove (2.3). (2.4) can be similarly obtained.

Similarly to (2.5), it follows from (1.5c) that for $t \geq T$ we have

$$
x=0: \frac{\partial \widetilde{\widetilde{u}}}{\partial x}-\left(\sum_{j=1}^{N} b_{i j}\right) \widetilde{\widetilde{u}}=0, \quad i=1, \ldots, N,
$$

then for $t \geq T$ we get

$$
x=0:\left(\sum_{j=1}^{N} b_{i j}\right) \widetilde{\widetilde{u}}=\left(\sum_{j=1}^{N} b_{k j}\right) \widetilde{\widetilde{u}}, \quad i, k=1, \ldots, N .
$$

Since the system is not exactly null controllable, we claim that at least for an initial data $(\Phi, \Psi)$, $\widetilde{\widetilde{u}}$ does not identically vanish at $x=0$ for $t \geq T$, then (2.3) holds. Otherwise, noting (2.7) we have

$$
x=0: \quad \widetilde{\widetilde{u}} \equiv \frac{\partial \widetilde{\widetilde{u}}}{\partial x} \equiv 0, \quad t \geq T
$$

then, by the exact boundary observability $[9,10]$ for the wave equation $(2.6)$, $\widetilde{u}$ should identically vanish for $t \geq T$ and $0 \leq x \leq L$, which gives a contradiction to the non exact null controllability.

This completes the proof of Lemma 2.1.

Remark 2.3. Conditions (2.2)-(2.4) mean that all the matrices $A, B$ and $\bar{B}$ have a common right eigenvector $(1,1, \ldots, 1)^{T}$ with the corresponding eigenvalues $\widetilde{\widetilde{a}}, \widetilde{\widetilde{b}}$ and $\widetilde{\bar{b}}$, respectively.

Theorem 2.4. Suppose that (2.2)-(2.4) hold and

$$
T>2 L \text {. }
$$

For any given initial state $(\Phi, \Psi) \in\left(C^{2}[0, L]\right)^{N} \times\left(C^{1}[0, L]\right)^{N}$, satisfying the conditions of $C^{2}$ compatibility at the point $(t, x)=(0, L)$, there exist $(N-1) C^{2}$ (case $\left.(1.5 \mathrm{a})\right)$ or $C^{1}$ (cases $\left.(1.5 \mathrm{~b})-(1.5 \mathrm{c})\right)$ boundary controls with support on $[0, T]$ in $H(t)$ (for example, take $h_{2}(t), \ldots, h_{N}(t)$ with $h_{1}(t) \equiv 0$ ), such that the coupled system (1.2), (1.5) and (1.7) possesses the exact boundary synchronization. 
Proof. Let

$$
w_{i}=u_{i}-u_{i+1} \quad(i=1, \ldots, N-1) .
$$

It is easily shown (see [13]) that by (2.2), the original system (1.2) for the variable $U$ can be reduced to a self-closing system of the same kind for the variable $W=\left(w_{1}, \ldots, w_{N-1}\right)^{T}$ as follows:

$$
\frac{\partial^{2} W}{\partial t^{2}}-\frac{\partial^{2} W}{\partial x^{2}}+\widetilde{A} W=0
$$

where $\widetilde{A}=\left(\widetilde{a}_{i j}\right)$ is an $(N-1) \times(N-1)$ matrix with the entries

$$
\widetilde{a}_{i j}=\sum_{p=j+1}^{N}\left(a_{i p}-a_{i+1, p}\right)=\sum_{p=1}^{j}\left(a_{i+1, p}-a_{i p}\right), \quad i, j=1, \ldots, N-1 .
$$

Similarly, by (2.3)-(2.4), the original boundary conditions (1.5) and (1.7) for the variable $U$ can be reduced, respectively, to self-closing boundary conditions of the same kind for the variable $W$ as follows:

$$
\begin{aligned}
& x=0: W=\widetilde{H}(t), \\
& x=0: W_{x}=\widetilde{H}(t), \\
& x=0: W_{x}-\widetilde{B} W=\widetilde{H}(t)
\end{aligned}
$$

and

$$
\begin{aligned}
& x=L: \quad W=0, \\
& x=L: W_{x}=0, \\
& x=L: \quad W_{x}+\widetilde{\bar{B}} W=0,
\end{aligned}
$$

where $\widetilde{B}=\left(\widetilde{b}_{i j}\right)$ and $\widetilde{\bar{B}}=\left(\widetilde{\bar{b}}_{i j}\right)$ are $(N-1) \times(N-1)$ coupling matrices reduced by $B=\left(b_{i j}\right)$ and $\bar{B}=\left(\bar{b}_{i j}\right)$ in the following way:

$$
\widetilde{b}_{i j}=\sum_{p=j+1}^{N}\left(b_{i p}-b_{i+1, p}\right)=\sum_{p=1}^{j}\left(b_{i+1, p}-b_{i p}\right), \quad i, j=1, \ldots, N-1
$$

etc. Moreover,

$$
\widetilde{H}(t)=\left(\widetilde{h}_{1}(t), \ldots, \widetilde{h}_{N-1}(t)\right)^{T}
$$

with

$$
\widetilde{h}_{i}(t)=h_{i}(t)-h_{i+1}(t), \quad i=1, \ldots, N-1 .
$$

The initial condition of $W$ is given by

$$
t=0: W=\widetilde{\Phi}(x), \quad W_{t}=\widetilde{\Psi}(x)
$$

with

$$
\left\{\begin{array}{l}
\widetilde{\Phi}(x)=\left(\varphi_{1}(x)-\varphi_{2}(x), \varphi_{2}(x)-\varphi_{3}(x), \ldots, \varphi_{N-1}(x)-\varphi_{N}(x)\right)^{T}, \\
\widetilde{\Psi}(x)=\left(\psi_{1}(x)-\psi_{2}(x), \psi_{2}(x)-\psi_{3}(x), \ldots, \psi_{N-1}(x)-\psi_{N}(x)\right)^{T} .
\end{array}\right.
$$

Thus, according to Lemma 1.6, by means of $(N-1) C^{2}$ (case $\left.(2.12 \mathrm{a})\right)$ or $C^{1}$ (cases $(2.12 \mathrm{~b})-(2.12 \mathrm{c})$ ) boundary controls $\widetilde{H}(t)$ (for instance, take $h_{2}(t), \ldots, h_{n}(t)$ with $h_{1}(t) \equiv 0$ ) at the end $x=0$, the variable $W$ is exactly null controllable, then the variable $U$ is exactly synchronizable. This finished the proof. 
Remark 2.5. Noting Remark 1.4, by Lemmas 1.1 and 1.6, the reduced mixed initial-boundary value problem (2.10), (2.12)-(2.13) and (2.17) for the variable $W$ is always well-posed and exactly null controllable.

Remark 2.6. As $t \geq T$, the synchronizable state $\widetilde{\widetilde{u}}=\widetilde{\widetilde{u}}(t, x)$ defined by (2.1) satisfies the wave equation (2.6) with the following boundary conditions:

$$
\begin{array}{ll}
x=0: & \widetilde{\widetilde{u}}=0, \\
x=0: & \widetilde{\widetilde{u}}_{x}=0, \\
x=0: & \widetilde{\widetilde{u}}_{x}-\widetilde{\widetilde{b}} \widetilde{u}=0
\end{array}
$$

and

$$
\begin{aligned}
& x=L: \quad \widetilde{\widetilde{u}}=0, \\
& x=L: \widetilde{\widetilde{u}}_{x}=0, \\
& x=L: \quad \widetilde{\widetilde{u}}_{x}+\widetilde{\widetilde{b}} \widetilde{\widetilde{u}}=0,
\end{aligned}
$$

where $\widetilde{\widetilde{a}}, \widetilde{\widetilde{b}}$ and $\widetilde{\bar{b}}$ are given by $(2.2)-(2.4)$, respectively.

Noting Remark 1.4, the forward mixed initial-boundary value problem for (2.6) and (2.19)-(2.20) is always well-posed. Hence, if we know the initial state of $\widetilde{\widetilde{u}}=\widetilde{\widetilde{u}}(t, x)$ at the moment $t=T$ :

$$
t=T: \widetilde{\widetilde{u}}=\varphi(x), \widetilde{\widetilde{u}}_{t}=\psi(x), \quad 0 \leq x \leq L,
$$

we can completely determine the evolution of $\widetilde{\widetilde{u}}=\widetilde{\widetilde{u}}(t, x)$ with respect to $t$.

Remark 2.7. Under assumptions (2.2)-(2.4), in order to realize the exact boundary synchronization, the $(N-$ 1) boundary controls in $H(t)$ can be chosen in infinitely many ways, therefore, the initial state $(\varphi, \psi)$ in $(2.21)$ will be not unique. Moreover, it can be shown that the attainable set of all possible initial data of synchronizable state is the whole space $C^{2}[0, L] \times C^{1}[0, L]$ associated with the conditions of $C^{2}$ compatibility at $(t, x)=(T, 0)$ and $(T, L)$, respectively, with boundary conditions $(2.19)$ and $(2.20)$.

To illustrate this, for any given $(\varphi, \psi) \in C^{2}[0, L] \times\left[C^{1}[0, L]\right.$ satisfying the conditions of $C^{2}$ compatibility with the boundary conditions $(2.19)-(2.20)$ at $(t, x)=(T, 0)$ and $(T, L)$ respectively, we can solve the backward mixed initial-boundary value problem (2.6) and (2.19)-(2.21) to get a unique $C^{2}$ solution $u=u(t, x)$ and its initial value at $t=0$

$$
t=0: u=\widehat{\varphi}(x), u_{t}=\widehat{\psi}(x) .
$$

Then, by assumptions (2.2)-(2.4), it is easy to see that

$$
U(t, x)=(u, u, \ldots, u)^{T}(t, x)
$$

is the $C^{2}$ solution to the original problem (1.2), (1.5) and (1.7) with the null control $H \equiv 0$ and the initial condition

$$
\begin{aligned}
t=0: U & =\widehat{\Phi}(x)=(\widehat{\varphi}(x), \widehat{\varphi}(x), \ldots, \widehat{\varphi}(x))^{T}, \\
U_{t} & =\widehat{\Psi}(x)=(\widehat{\psi}(x), \widehat{\psi}(x), \ldots, \widehat{\psi}(x))^{T} .
\end{aligned}
$$

Then, by solving the mixed problem (1.2), (1.5), (1.7) and (2.24) with null boundary controls, we can reach any given synchronizable state $(\varphi, \psi)$ at the moment $t=T$. 


\section{EXACT BOUNDARY SYNCHRONIZATION BY GROUPS}

The concept of exact boundary synchronization can be generalized to the exact boundary synchronization by groups. Without loss of generality, we consider here the exact boundary synchronization by 2-groups. For this purpose, setting $U=\left(\begin{array}{c}U^{(1)} \\ U^{(2)}\end{array}\right)$ with $U^{(1)}=\left(u_{1}, \ldots, u_{m}\right)^{T}$ and $U^{(2)}=\left(u_{m+1}, \ldots, u_{N}\right)^{T}$, we look for some boundary controls in $H(t)$, such that $U^{(1)}$ and $U^{(2)}$ are independently synchronizable.

Definition 3.1. If there exists $T>0$ such that for any given initial state $(\Phi(x), \Psi(x))$, we can find some boundary controls with support on $[0, T]$ in $H(t)$, such that the corresponding mixed initial-boundary value problem (1.2), (1.5), (1.7) and (1.8) admits a unique $C^{2}$ solution $U=U(t, x)=\left(u_{1}(t, x), \ldots, u_{N}(t, x)\right)^{T}$ on $t \geq 0$, and for $t \geq T$ we have

$$
\begin{aligned}
u_{1}(t, x) \equiv \ldots \equiv u_{m}(t, x) \stackrel{\text { def. }}{=} \widetilde{\widetilde{u}}_{1}(t, x), & 0 \leq x \leq L, \\
u_{m+1}(t, x) \equiv \ldots \equiv u_{N}(t, x) \stackrel{\text { def. }}{=} \widetilde{\widetilde{u}}_{2}(t, x), & 0 \leq x \leq L,
\end{aligned}
$$

then the coupled system (1.2), (1.5) and (1.7) is said to possess the exact boundary synchronization by 2-groups or is said to be exactly synchronizable by 2-groups, and $\widetilde{\widetilde{U}}=\widetilde{\widetilde{U}}(t, x)=\left(\begin{array}{c}\tilde{\tilde{u}}_{1}(t, x) \\ \tilde{u}_{2}(t, x)\end{array}\right)$ is called the corresponding synchronizable state by 2 -groups.

Obviously, if the coupled system possesses the exact boundary synchronization, then it must be exactly synchronizable by 2 -groups. However, this trivial situation should be excluded in advance. For this purpose we need to impose suitable assumptions on the synchronizable states by 2 -groups $\widetilde{\widetilde{u}}_{1}(t, x)$ and $\widetilde{\widetilde{u}}_{2}(t, x)$. We have

Lemma 3.2. Suppose that the coupled system (1.2), (1.5) and (1.7) is exactly synchronizable by 2-groups. Suppose furthermore that at least for an initial state $(\Phi(x), \Psi(x))$ the synchronizable states by 2-groups $\widetilde{\widetilde{u}}_{1}(t, x)$ and $\widetilde{\widetilde{u}}_{2}(t, x)$ are linearly independent. Then the coupling matrix $A=\left(a_{i j}\right)$ in system (1.2) of wave equations should satisfy the following necessary conditions:

$$
\begin{gathered}
\sum_{j=1}^{m} a_{i j} \stackrel{\text { def. }}{=} \begin{cases}\widetilde{\widetilde{a}}_{11} & (i=1, \ldots, m), \\
\widetilde{\widetilde{a}}_{21} & (i=m+1, \ldots, N),\end{cases} \\
\sum_{j=m+1}^{N} a_{i j} \stackrel{\text { def. }}{=} \begin{cases}\widetilde{\widetilde{a}}_{12} & (i=1, \ldots, m), \\
\widetilde{\widetilde{a}}_{22} & (i=m+1, \ldots, N),\end{cases}
\end{gathered}
$$

where $\widetilde{\widetilde{a}}_{11}$ and $\widetilde{\widetilde{a}}_{12}$ are independent of $i=1, \ldots, m$, while, $\widetilde{\widetilde{a}}_{21}$ and $\widetilde{\widetilde{a}}_{22}$ are independent of $i=m+1, \ldots, N$.

Proof. By Definition 3.1, for $t \geq T$ we have

$$
\begin{aligned}
& \frac{\partial^{2} \widetilde{\widetilde{u}}_{1}}{\partial t^{2}}-\frac{\partial^{2} \widetilde{\widetilde{u}}_{1}}{\partial x^{2}}+\left(\sum_{p=1}^{m} a_{i p}\right) \widetilde{\widetilde{u}}_{1}+\left(\sum_{p=m+1}^{N} a_{i p}\right) \widetilde{\widetilde{u}}_{2}=0, \quad 0 \leq x \leq L, i=1, \ldots, m, \\
& \frac{\partial^{2} \widetilde{\widetilde{u}}_{2}}{\partial t^{2}}-\frac{\partial^{2} \widetilde{\widetilde{u}}_{2}}{\partial x^{2}}+\left(\sum_{p=1}^{m} a_{i p}\right) \widetilde{\widetilde{u}}_{1}+\left(\sum_{p=m+1}^{N} a_{i p}\right) \widetilde{\widetilde{u}}_{2}=0, \quad 0 \leq x \leq L, i=m+1, \ldots, N .
\end{aligned}
$$

Hence we get

$$
t \geq T:\left(\sum_{p=1}^{m} a_{i p}-\sum_{p=1}^{m} a_{k p}\right) \widetilde{\widetilde{u}}_{1}+\left(\sum_{p=m+1}^{N} a_{i p}-\sum_{p=m+1}^{N} a_{k p}\right) \widetilde{\widetilde{u}}_{2}=0, \quad 0 \leq x \leq L
$$


for $i, k=1, \ldots, m$ and $i, k=m+1, \ldots, N$, respectively. Since at least for an initial data $(\Phi(x), \Psi(x))$, the synchronizable states by 2-groups, $\widetilde{\widetilde{u}}_{1}$ and $\widetilde{\widetilde{u}}_{2}$ are linearly independent, we get (3.3)-(3.4) immediately.

For the coupling matrices $B$ and $\bar{B}$, we can not get the corresponding necessary conditions under the assumptions of Lemma 3.2 (unless we assume that at least for an initial data $(\Phi(x), \Psi(x))$, $\widetilde{\widetilde{u}}_{1}(t, x)$ and $\widetilde{\widetilde{u}}_{2}(t, x)$ are linearly independent at $x=0$ and at $x=L$, respectively). However, based on Lemma 3.2, in what follows we impose the following similar assumptions:

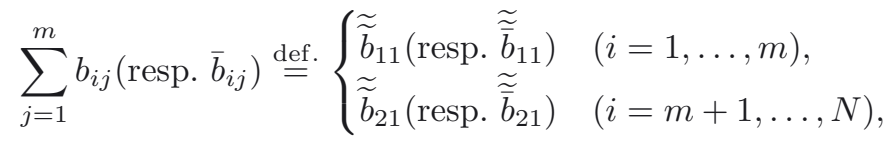

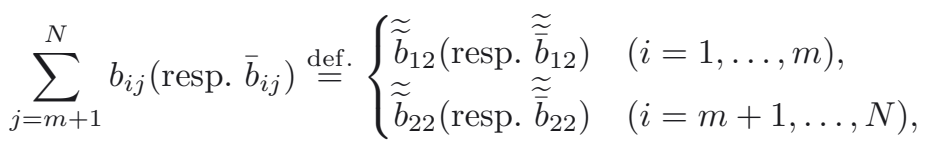

$\underset{\text { where }}{ } \widetilde{\widetilde{b}}_{11}$ (resp. $\widetilde{\widetilde{b}}_{11}$ ) and $\widetilde{\widetilde{b}}_{12}$ (resp. $\widetilde{\widetilde{b}}_{12}$ ) are independent of $i=1, \ldots, m$, while, $\widetilde{\widetilde{b}}_{21}$ (resp. $\widetilde{\widetilde{b}}_{21}$ ) and $\widetilde{\widetilde{b}}_{22}$ (resp. $\widetilde{\widetilde{b}}_{22}$ ) are independent of $i=m+1, \ldots, N$.

Theorem 3.3. Suppose that (3.3)-(3.4) and (3.7)-(3.8) hold and $T>0$ satisfies (2.8). For any given initial state $(\Phi(x), \Psi(x))$, there exist $(N-2) C^{2}$ (case (1.5a)) or $C^{1}$ (cases $(1.5 \mathrm{~b})-(1.5 \mathrm{c})$ ) boundary controls with support on $[0, T]$ in $H(t)$ (for instance, $h_{2}(t), \ldots, h_{m}(t), h_{m+2}(t), \ldots, h_{N}(t)$ with $\left.h_{1}(t) \equiv h_{m+1}(t) \equiv 0\right)$, such that the coupled system (1.2), (1.5) and (1.7) possesses the exact boundary synchronization by 2-groups.

Proof. Let

$$
\left\{\begin{array}{l}
w_{i}=u_{i}-u_{i+1} \quad(i=1, \ldots, m-1) \\
w_{i}=u_{i+1}-u_{i+2} \quad(i=m, \ldots, N-2) .
\end{array}\right.
$$

Similarly to the proof of Theorem 2.4 (cf. [13]), under assumptions (3.3)-(3.4), the original system (1.2) for the variable $U$ can be reduced to a self-closing system (2.10) of the same kind for the variable $W=\left(w_{1}, \ldots, w_{N-2}\right)^{T}$, where $\widetilde{A}=\left(\widetilde{a}_{i j}\right)$ is an $(N-2) \times(N-2)$ matrix with the entries

$$
\widetilde{a}_{i j}=\left\{\begin{array}{l}
\sum_{p=j+1}^{m}\left(a_{i p}-a_{i+1, p}\right)=\sum_{p=1}^{j}\left(a_{i+1, p}-a_{i p}\right), \quad i=1, \ldots, N-2 ; j=1, \ldots, m-1, \\
\sum_{p=j+2}^{N}\left(a_{i p}-a_{i+1, p}\right)=\sum_{p=m+1}^{j+1}\left(a_{i+1, p}-a_{i p}\right), \quad i=1, \ldots, N-2 ; j=m, \ldots, N-2 .
\end{array}\right.
$$

Similarly, under assumptions (3.7)-(3.8), the original boundary conditions (1.5) and (1.7) for the variable $U$ can be reduced to self-closing boundary conditions (2.12) and (2.13) of the same kind for the variable $W$, in which the coupling matrices $\widetilde{B}=\left(\widetilde{b}_{i j}\right)$ and $\widetilde{\bar{B}}=\left(\widetilde{\bar{b}}_{i j}\right)$ are $(N-2) \times(N-2)$ matrices reduced by $B=\left(b_{i j}\right)$ and $\bar{B}=\left(\bar{b}_{i j}\right)$ in the following way:

$$
\widetilde{b}_{i j}=\left\{\begin{array}{l}
\sum_{p=j+1}^{m}\left(b_{i p}-b_{i+1, p}\right)=\sum_{p=1}^{j}\left(b_{i+1, p}-b_{i p}\right), \quad i=1, \ldots, N-2 ; j=1, \ldots, m-1, \\
\sum_{p=j+2}^{N}\left(b_{i p}-b_{i+1, p}\right)=\sum_{p=m+1}^{j+1}\left(b_{i+1, p}-b_{i p}\right), \quad i=1, \ldots, N-2 ; j=m, \ldots, N-2
\end{array}\right.
$$

etc. Moreover,

$$
\widetilde{H}(t)=\left(\widetilde{h}_{1}(t), \ldots, \widetilde{h}_{N-2}(t)\right)
$$


with

$$
\widetilde{h}_{i}(t)=\left\{\begin{array}{l}
h_{i}(t)-h_{i+1}(t), \quad i=1, \ldots, m-1, \\
h_{i+1}(t)-h_{i+2}(t), \quad i=m, \ldots, N-2 .
\end{array}\right.
$$

The initial condition $(2.17)$ of $W$ is given by

$$
\widetilde{\Phi}(x)=\left(\widetilde{\varphi}_{1}(x), \ldots, \widetilde{\varphi}_{N-2}(x)\right)^{T}, \quad \widetilde{\Psi}(x)=\left(\widetilde{\psi}_{1}(x), \ldots, \widetilde{\psi}_{N-2}(x)\right)^{T}
$$

with

$$
\begin{aligned}
& \widetilde{\varphi}_{i}(x)=\left\{\begin{array}{l}
\varphi_{i}(x)-\varphi_{i+1}(x), \quad i=1, \ldots, m-1, \\
\varphi_{i+1}(x)-\varphi_{i+2}(x), \quad i=m, \ldots, N-2,
\end{array}\right. \\
& \widetilde{\Psi}_{i}(x)=\left\{\begin{array}{l}
\psi_{i}(x)-\psi_{i+1}(x), \quad i=1, \ldots, m-1, \\
\psi_{i+1}(x)-\psi_{i+2}(x), \quad i=m, \ldots, N-2 .
\end{array}\right.
\end{aligned}
$$

Thus, according to Lemma 1.6, by means of $(N-2) C^{2}$ (case (2.12a)) or $C^{1}$ (cases $\left.(2.12 \mathrm{~b})-(2.12 \mathrm{c})\right)$ boundary controls $\widetilde{H}(t)$ (for instance, take $h_{2}(t), \ldots, h_{m}(t), h_{m+2}(t), \ldots, h_{N}(t)$ with $\left.h_{1}(t) \equiv h_{m+1}(t) \equiv 0\right)$ at the end $x=0$, the variable $W$ is exactly null controllable, then the variable $U$ is exactly synchronizable by 2-groups. This completes the proof.

Remark 3.4. Lemmas 1.1 and 1.6 are always available for the reduced mixed initial-boundary value problem (2.10), (2.12)-(2.13) and (3.14) for the variable $W$.

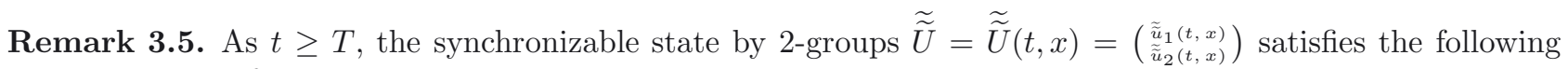
coupled system of wave equations

$$
\frac{\partial^{2} \widetilde{\widetilde{U}}}{\partial t^{2}}-\frac{\partial^{2} \widetilde{\widetilde{U}}}{\partial x^{2}}+\widetilde{\widetilde{A}} \widetilde{\widetilde{U}}=0
$$

where

$$
\widetilde{\widetilde{A}}=\left(\begin{array}{ll}
\widetilde{\widetilde{a}}_{11} & \widetilde{\widetilde{a}}_{12} \\
\widetilde{\widetilde{a}}_{21} & \widetilde{\widetilde{a}}_{22}
\end{array}\right),
$$

and $\widetilde{\widetilde{a}}_{11}, \widetilde{\widetilde{a}}_{12}, \widetilde{\widetilde{a}}_{21}$ and $\widetilde{\widetilde{a}}_{21}$ are given by $(3.3)-(3.4)$. Moreover, $\widetilde{\widetilde{U}}=\widetilde{\widetilde{U}}(t, x)$ satisfies the following boundary conditions:

$$
\begin{array}{ll}
x=0: & \widetilde{\widetilde{U}}=0, \\
x=0: & \widetilde{\widetilde{U}}_{x}=0, \\
x=0: & \widetilde{\widetilde{U}}_{x}-\widetilde{\widetilde{B}} \widetilde{\widetilde{U}}=0
\end{array}
$$

and

$$
\begin{array}{ll}
x=0: & \widetilde{\widetilde{U}}=0, \\
x=0: & \widetilde{\widetilde{U}}_{x}=0, \\
x=0: & \widetilde{\widetilde{U}}_{x}+\widetilde{\widetilde{B}} \widetilde{\widetilde{U}}=0,
\end{array}
$$


where

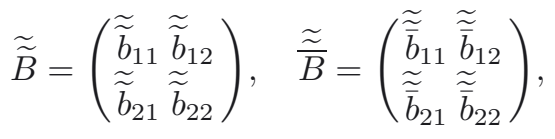

the elements of which are provided by (3.7)-(3.8).

Hence, by Lemma 1.1, if we know the initial state of $\widetilde{\widetilde{U}}=\widetilde{\widetilde{U}}(t, x)$ at the moment $t=T$ :

$$
t=T: \widetilde{\widetilde{U}}=\left(\varphi^{(1)}(x), \varphi^{(2)}(x)\right)^{T}, \widetilde{\widetilde{U}}_{t}=\left(\psi^{(1)}(x), \psi^{(2)}(x)\right)^{T}, \quad 0 \leq x \leq L,
$$

then the evolution of $\widetilde{\widetilde{U}}=\widetilde{\widetilde{U}}(t, x)$ with respect to $t$ can be completely determined.

Remark 3.6. Under assumptions (3.3)-(3.4) and (3.7)-(3.8), in order to realize the exact boundary synchronization by 2 -groups, one has infinitively many ways to choose $(N-2)$ boundary controls at the end $x=0$. Moreover, as in Remark 2.7, any given state $\left(\varphi^{(1)}, \varphi^{(2)}\right)^{T}$ and $\left(\psi^{(1)}, \psi^{(2)}\right)^{T}$ in $\left(C^{2}[0, L]\right)^{2} \times\left(C^{1}[0, L]\right)^{2}$, satisfying the conditions of $C^{2}$ compatibility at the points $(t, x)=(T, 0)$ and $(T, L)$, respectively, with boundary conditions (3.18) and (3.19), belongs to the attainable set of the initial data of synchronizable state by 2 -groups.

Thus, at least for some initial states $(\Phi(x), \Psi(x))$, the synchronizable states by 2-groups $\widetilde{\widetilde{u}}_{1}(t, x)$ and $\widetilde{\widetilde{u}}_{2}(t, x)$ are linearly independent on $t=T$ then for $t \geq T$. It shows that the corresponding requirement on $\widetilde{\widetilde{u}}_{1}$ and $\widetilde{\widetilde{u}}_{2}$, given in Lemma 3.1, is reasonable.

Remark 3.7. In the special case that $m=N-1, U^{(2)}(t, x)$ is composed of only one component $u_{N}(t, x)$, then assumptions (3.4) and (3.8) reduce to

$$
a_{i N}=\widetilde{\widetilde{a}}_{12} \quad(i=1, \ldots, N-1),
$$

and

$$
b_{i N}\left(\operatorname{resp} . \bar{b}_{i N}\right)=\widetilde{\widetilde{b}}_{12}\left(\operatorname{resp} . \widetilde{\bar{b}}_{12}\right) \quad(i=1, \ldots, N-1),
$$

where $\widetilde{\widetilde{a}}_{12}$ and $\widetilde{\widetilde{b}}_{12}$ (resp. $\widetilde{\widetilde{b}}_{12}$ ) are independent of $i=1, \ldots, N-1$, namely, the first $(N-1)$ components of the last column of the coupling matrices $A$ and $B$ (resp. $\bar{B}$ ) are the same, respectively.

Remark 3.8. The exact boundary synchronization by $k$-groups can be treated in a similar way.

In order to realize the exact boundary synchronization by 2-groups, the number of boundary controls is equal to $(N-2)$, while, in order to realize the exact boundary synchronization by $k$-groups, the number of boundary controls is equal to $(N-k)$.

\section{EXACT BOUNDARY NULL CONTROLLABILITY AND SYNCHRONIZATION BY GROUPS}

For the coupled system of wave equations (1.2), (1.5) and (1.7), by Lemma 1.6, we can use $N$ boundary controls to realize the exact boundary null controllability. When the number of boundary controls is less than $N$, however, generically speaking, it is impossible to realize the same requirement. A natural question is whether we can get the exact boundary null controllability for a part of state variables when the number of boundary controls is less than $N$. For instance, is it possible or not to realize the exact boundary null controllability for $(N-1)$ state variables by means of only $(N-1)$ boundary controls? Since all the state variables are coupled each other, generally speaking, this requirement is impossible to be realized. However, following the idea given in Section 3, under certain assumptions on the coupled system, if we divide the state variables into two groups $U^{(1)}=\left(u_{1}, \ldots, u_{m}\right)^{T}$ and $U^{(2)}=\left(u_{m+1}, \ldots, u_{N}\right)^{T}$ such that $U^{(1)}$ is exactly null controllable, while $U^{(2)}$ is exactly synchronizable, then we can realize the previous requirement in some sense. It leads to the following 
Definition 4.1. If there exists $T>0$ such that for any given initial state $(\Phi(x), \Psi(x))$, we can find some boundary controls with support on $[0, T]$ in $H(t)$, such that the corresponding mixed initial-boundary value problem (1.2), (1.5), (1.7) and (1.8) admits a unique $C^{2}$ solution $U=U(t, x)=\left(u_{1}(t, x), \ldots, u_{N}(t, x)\right)^{T}$ on $t \geq 0$, and as $t \geq T$ we have

$$
\begin{aligned}
u_{1}(t, x) & \equiv \ldots \equiv u_{m}(t, x) \equiv 0, \quad 0 \leq x \leq L, \\
u_{m+1}(t, x) & \equiv \ldots \equiv u_{N}(t, x) \stackrel{\text { def. }}{=} \widetilde{\widetilde{u}}(t, x), \quad 0 \leq x \leq L,
\end{aligned}
$$

then the coupled system (1.2), (1.5) and (1.7) is said to possess the exact boundary null controllability and

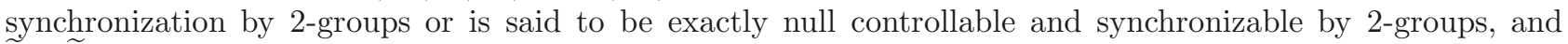
$\widetilde{\widetilde{u}}=\widetilde{\widetilde{u}}(t, x)$ is called to be the partially synchronizable state.

Obviously, if the coupled system (1.2), (1.5) and (1.7) possesses the exact null controllability, then it must satisfy the requirement of Definition 4.1. This situation should be excluded in advance.

Lemma 4.2. Suppose that the coupled system (1.2), (1.5) and (1.7) is exactly null controllable and synchronizable by 2-groups, but not exactly null controllable, then the coupling matrix $A=\left(a_{i j}\right)$ in system (1.2) must satisfy the following necessary conditions:

$$
\begin{aligned}
& \sum_{j=m+1}^{N} a_{i j}=0 \quad(i=1, \ldots, m), \\
& \sum_{j=m+1}^{N} a_{i j} \stackrel{\text { def. }}{=} \widetilde{\widetilde{a}}(i=m+1, \ldots, N),
\end{aligned}
$$

where $\widetilde{\widetilde{a}}$ is a constant independent of $i=m+1, \ldots, N$.

For the coupling matrices $B=\left(b_{i j}\right)$ and $\bar{B}=\left(\bar{b}_{i j}\right)$ in the boundary conditions (1.5) and (1.7), similar necessary conditions hold, namely,

$$
\begin{aligned}
& \sum_{j=m+1}^{N} b_{i j}\left(\text { resp. } \bar{b}_{i j}\right)=0 \quad(i=1, \ldots, m), \\
& \sum_{j=m+1}^{N} b_{i j}\left(\text { resp. } \bar{b}_{i j}\right) \stackrel{\text { def. }}{=} \widetilde{\widetilde{b}}(\operatorname{resp} . \widetilde{\widetilde{b}}) \quad(i=m+1, \ldots, N),
\end{aligned}
$$

where $\widetilde{\widetilde{b}}$ and $\widetilde{\widetilde{b}}$ are constants independent of $i=m+1, \ldots, N$.

Proof. By Definition 4.1, it follows from (1.4) that for $t \geq T$ we have

$$
\begin{aligned}
& \left(\sum_{j=m+1}^{N} a_{i j}\right) \widetilde{\widetilde{u}}=0, \quad 0 \leq x \leq L, i=1, \ldots, m, \\
& \frac{\partial^{2} \widetilde{\widetilde{u}}}{\partial t^{2}}-\frac{\partial^{2} \widetilde{\widetilde{u}}}{\partial x^{2}}+\left(\sum_{j=m+1}^{N} a_{i j}\right) \widetilde{\widetilde{u}}=0, \quad 0 \leq x \leq L, i=m+1, \ldots, N .
\end{aligned}
$$

In particular, for $t \geq T$ we get from (4.8) that

$$
\left(\sum_{j=m+1}^{N} a_{i j}\right) \widetilde{\widetilde{u}}=\left(\sum_{j=m+1}^{N} a_{k j}\right) \widetilde{\widetilde{u}}, \quad 0 \leq x \leq L, i, k=m+1, \ldots, N .
$$

Then, (4.3)-(4.4) follow directly from the non exact null controllability. 
Similarly to the proof of Lemma 2.2, we get (4.5)-(4.6).

Theorem 4.3. Suppose that (4.3)-(4.6) hold and $T>0$ satisfies (2.8). For any given initial state $(\Phi(x), \Psi(x))$, we can find $(N-1) C^{2}$ (case $\left.(1.5 \mathrm{a})\right)$ or $C^{1}$ (cases $(1.5 \mathrm{~b})-(1.5 \mathrm{c})$ ) boundary controls with support on $[0, T]$ in $H(t)$ (for instance, take $h_{1}(t), \ldots, h_{m}(t), h_{m+2}(t), \ldots, h_{N}(t)$ with $\left.h_{m+1}(t) \equiv 0\right)$, such that the coupled system (1.2), (1.5) and (1.7) possesses the exact boundary null controllability and synchronization by 2-groups.

Proof. Let

$$
\left\{\begin{array}{l}
w_{i}=u_{i} \quad(i=1, \ldots, m) \\
w_{i}=u_{i}-u_{i+1} \quad(i=m+1, \ldots, N-1) .
\end{array}\right.
$$

Similarly to the proof of Theorem 2.4 (cf. [13]), under assumptions (4.3)-(4.4), the original system (1.2) for the variable $U$ can be reduced to a self-closing system $(2.10)$ of the same kind for the variable $W=\left(w_{1}, \ldots, w_{N-1}\right)^{T}$, where $\widetilde{A}=\left(\widetilde{a}_{i j}\right)$ is an $(N-1) \times(N-1)$ matrix with

$$
\begin{aligned}
& \widetilde{a}_{i j}=\left\{\begin{array}{l}
a_{i j}, \quad j=1, \ldots, m, \\
\sum_{p=j+1}^{N} a_{i p}, \quad j=m+1, \ldots, N-1,
\end{array} \text { for } i=1, \ldots, m,\right. \\
& \tilde{a}_{i j}=\left\{\begin{array}{cc}
a_{i j}-a_{i+1, j}, \quad j=1, \ldots, m, & \text { for } i=m+1, \ldots, N-1 . \\
\sum_{p=j+1}^{N}\left(a_{i p}-a_{i+1, p}\right)=\sum_{p=m+1}^{j}\left(a_{i+1, p}-a_{i p}\right), & j=m+1, \ldots, N-1,
\end{array}\right.
\end{aligned}
$$

Similarly, by (4.5)-(4.6), the original boundary conditions (1.5) and (1.7) for the variable $U$ can be reduced, respectively, to a self-closing boundary conditions $(2.12)$ and $(2.13)$ of the same kind for the variable $W$, in which the coupling matrices $\widetilde{B}=\left(\widetilde{b}_{i j}\right)$ and $\widetilde{\bar{B}}=\left(\widetilde{\bar{b}}_{i j}\right)$ are $(N-1) \times(N-1)$ matrices reduced by $B=\left(b_{i j}\right)$ and $\bar{B}=\left(\bar{b}_{i j}\right)$ in the following way:

$$
\begin{aligned}
& \widetilde{b}_{i j}=\left\{\begin{array}{lr}
b_{i j}, \quad j=1, \ldots, m, \\
\sum_{p=j+1}^{N} b_{i p}, \quad j=m+1, \ldots, N-1, & \text { for } i=1, \ldots, m,
\end{array}\right. \\
& \widetilde{b}_{i j}=\left\{\begin{array}{cc}
b_{i j}-b_{i+1, j}, \quad j=1, \ldots, m, & \text { for } i=m+1, \ldots, N-1 \\
\sum_{p=j+1}^{N}\left(b_{i p}-b_{i+1, p}\right)=\sum_{p=1}^{j}\left(b_{i+1, p}-b_{i p}\right), & j=m+1, \ldots, N-1,
\end{array}\right.
\end{aligned}
$$

etc. Moreover, $\widetilde{H}(t)$ is given by $(2.15)$ with

$$
\widetilde{h}_{i}(t)=\left\{\begin{array}{l}
h_{i}(t), \quad i=1, \ldots, m \\
h_{i}(t)-h_{i+1}(t), \quad i=m+1, \ldots, N-1 .
\end{array}\right.
$$

The initial condition $(2.17)$ of $W$ is given by

$$
\begin{aligned}
& \widetilde{\varphi}_{i}(x)=\left\{\begin{array}{l}
\varphi_{i}(x), \quad i=1, \ldots, m, \\
\varphi_{i}(x)-\varphi_{i+1}(x), \quad i=m+1, \ldots, N-1,
\end{array}\right. \\
& \widetilde{\psi}_{i}(x)=\left\{\begin{array}{l}
\psi_{i}(x), \quad i=1, \ldots, m, \\
\psi_{i}(x)-\psi_{i+1}(x), \quad i=m+1, \ldots, N-1 .
\end{array}\right.
\end{aligned}
$$


Thus, according to Lemma 1.6, by means of $(N-1) C^{2}$ (case (2.12a)) or $C^{1}$ (cases $\left.(2.12 \mathrm{~b})-(2.12 \mathrm{c})\right)$ boundary controls $\widetilde{H}(t)$ (for instance, take $h_{1}(t), \ldots, h_{m}(t), h_{m+1}(t), \ldots, h_{N}(t)$ with $h_{m+1}(t) \equiv 0$ ) at the end $x=0$, the variable $W$ is exactly null controllable, then the variable $U$ is exactly null controllable and synchronizable by 2-groups. This proves Theorem 4.3.

Remark 4.4. Lemmas 1.1 and 1.6 are always available for the reduced initial-boundary value problem for the variable $W$.

Remark 4.5. As $t \geq T$, the partially synchronizable state $\widetilde{\widetilde{u}}=\widetilde{\widetilde{u}}(t, x)$ satisfies wave equation $(2.6)$ and boundary conditions (2.19) and (2.20), where $\widetilde{\widetilde{a}}, \widetilde{\widetilde{b}}$ and $\widetilde{\bar{b}}$ are given by (4.4) and (4.6). Lemma 1.1 is always available for this system (2.6) and (2.19)-(2.20).

Hence, if we know this initial condition $(2.21)$ of $\widetilde{\widetilde{u}}=\widetilde{\widetilde{u}}(t, x)$ at the moment $t=T$, then the evolution of the partially synchronizable state $\widetilde{\widetilde{u}}=\widetilde{\widetilde{u}}(t, x)$ with respect to $t$ can be completely determined.

Moreover, any given state $(\varphi, \psi)$ in $C^{2}[0, L] \times C^{1}[0, L]$, satisfying the conditions of $C^{2}$ compatibility at the points $(t, x)=(T, 0)$ and $(T, L)$, respectively, with boundary conditions (2.19) and (2.20), belongs to the attainable set of the initial data of partially synchronizable state.

Remark 4.6. In the special case that $m=N-1, U^{(2)}(t, x)$ is composed of only one component $u_{N}(t, x)$, then assumptions (4.3) and (4.5) become

$$
\begin{aligned}
& a_{i N}=0 \quad(i=1, \ldots, N-1), \\
& b_{i N}\left(\text { resp. } \bar{b}_{i N}\right)=0 \quad(i=1, \ldots, N-1),
\end{aligned}
$$

namely, the first $(N-1)$ components of the last column of the coupling matrices $A$ and $B$ (resp. $\bar{B}$ ) are all zero. In this case, by Theorem $4.3,(N-1)$ boundary controls can be used to realize the exact boundary null controllability for the first $(N-1)$ state variables of $U$, however, since the first $(N-1)$ wave equations and boundary conditions constitute a self-closing system, this result is obvious.

Now, taking $m=N-2$ in Theorem 4.3, assumptions (4.3) and (4.6) become

$$
\begin{aligned}
& \sum_{j=N-1}^{N} a_{i j}=0 \quad(i=1, \ldots, N-2), \\
& \sum_{j=N-1}^{N} a_{i j}=\widetilde{\widetilde{a}} \quad(i=N-1, N)
\end{aligned}
$$

and

$$
\begin{aligned}
& \sum_{j=N-1}^{N} b_{i j}\left(\text { resp. } \bar{b}_{i j}\right)=0, \quad(i=1, \ldots, N-2), \\
& \sum_{j=N-1}^{N} b_{i j}\left(\operatorname{resp} . \bar{b}_{i j}\right)=\widetilde{\widetilde{b}}(\operatorname{resp} . \widetilde{\widetilde{b}}) \quad(i=N-1, N) .
\end{aligned}
$$

Thus, we can use $(N-1)$ (instead of $(N-2)$ !) boundary controls to realize the exact boundary null controllability for $(N-2)$ state variables in $U$. 


\section{Generalized EXACT BOUNDARY SYNCHRONIZATION}

We now consider the problem of synchronization from a more general mathematical point of view.

For the coupled system of wave equations (1.2), (1.5) and (1.7), based on the previous discussions, we can define the corresponding generalized exact boundary synchronization.

Let

$$
w_{i}=\sum_{j=1}^{N} \theta_{i j} u_{j} \quad(i=1, \ldots, M)
$$

or

$$
W=\mathrm{H} U \text {, }
$$

where $W=\left(w_{1}, \ldots, w_{M}\right)^{T}, 0<M<N$ and $\mathrm{H}=\left(\theta_{i j}\right)_{M \times N}$ is of full row-rank.

Suppose that for any given initial state $(\Phi(x), \Psi(x))$, the mixed initial-boundary value problem (1.2), (1.5), (1.7) and (1.8) for the variable $U$ can be reduced to a self-closing mixed initial-boundary values problem of the same kind for the variable $W$, and we can use Lemma 1.6 to find $M C^{2}$ (case (1.5a)) or $C^{1}$ (cases $\left.(1.5 \mathrm{~b})-(1.5 \mathrm{c})\right)$ boundary controls in $H(t)$ with support on $[0, T]$ with $T>2 L$, such that as $t \geq T$ we have

$$
W(t, x) \equiv 0, \quad 0 \leq x \leq L,
$$

i.e.,

$$
\text { (H) } U(t, x) \equiv 0, \quad 0 \leq x \leq L \text {. }
$$

Thus, the coupled system $(1.2),(1.5)$ and (1.7) is said to have the generalized exact boundary synchronization associated with $\mathrm{H}$ and $U=U(t, x)$ is the corresponding generalized synchronizable state.

It is easily seen that this definition covers all the cases discussed in the previous sections.

(1) If $M=N-1$ and we take

$$
w_{i}=u_{i}-u_{i+1} \quad(i=1, \ldots, N-1),
$$

i.e.,

$$
\mathrm{H}=\left(\begin{array}{cccc}
1 & -1 & & \\
& \ddots & \ddots & \\
& & 1 & -1
\end{array}\right)_{(N-1) \times N},
$$

then the generalized exact synchronization leads to the exact synchronization given in Section 2 .

(2) If $M=N-1$ and we take

$$
\left\{\begin{array}{l}
w_{i}=u_{i} \quad(i=1, \ldots, m), \\
w_{i}=u_{i}-u_{i+1} \quad(i=m+1, \ldots, N-1),
\end{array}\right.
$$

i.e.,

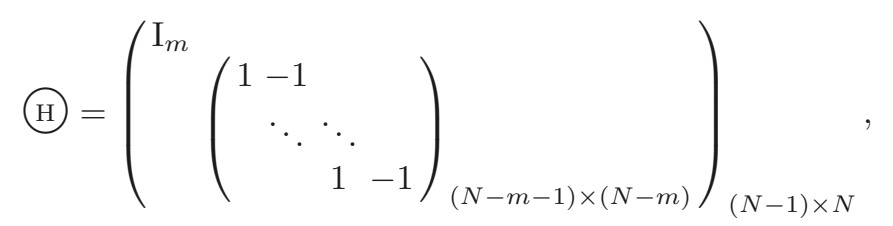

where $\mathrm{I}_{m}$ is the unit matrix of order $m$, then the generalized exact synchronization leads to the exact null controllability and synchronization by 2 -groups discussed in Section 4 . 
(3) If $M=N-2$ and we take

$$
\left\{\begin{array}{l}
w_{i}=u_{i}-u_{i+1} \quad(i=1, \ldots, m-1) \\
w_{i}=u_{i+1}-u_{i+2} \quad(i=m, \ldots, N-2),
\end{array}\right.
$$

i.e.,

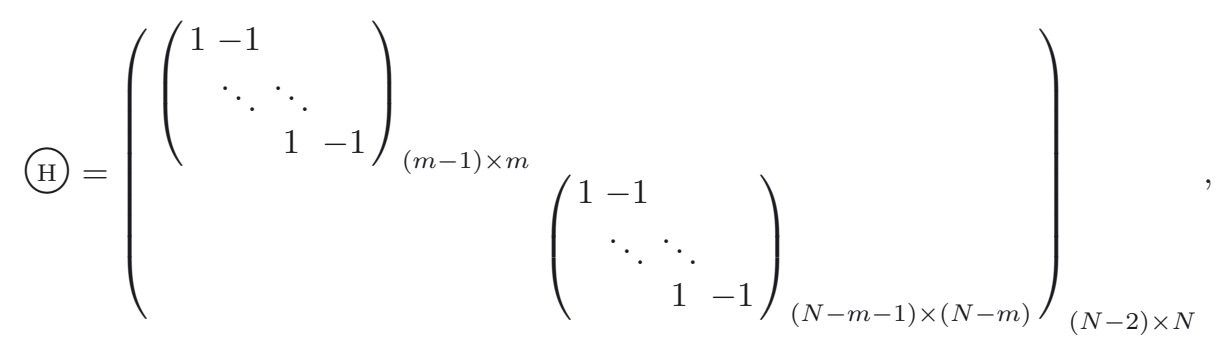

then the generalized exact synchronization leads to the exact synchronization by 2-groups discussed in Section 3 .

A natural question is under what conditions the original coupled system for the variable $U$ can be reduced to a self-closing coupled system of the same type for the variable $W$.

Multiplying the matrix $(H)$ to the system (1.2), (1.5) and (1.7) from the left, it is easy to get

Theorem 5.1. For any given coupling $N \times N$ matrices $A, B$ and $\bar{B}$, if there exist a full row-rank matrix H $=\left(\theta_{i j}\right)_{M \times N}$, and $M \times M$ matrices $\widetilde{A}, \widetilde{B}$ and $\widetilde{\bar{B}}$ such that

$$
\begin{aligned}
& \text { Н } A=\widetilde{A} \text { Н }, \\
& \text { Н } B=\widetilde{B} \mathrm{H}, \\
& \text { Н } \bar{B}=\widetilde{\bar{B}} \mathrm{H},
\end{aligned}
$$

then we can get a self-closing coupled system (2.10) and (2.12)-(2.13) with $\widetilde{H}(t)=\mathrm{H} H(t)$ for the variable $W$, so that the generalized exact boundary synchronization associated with $\mathrm{H}$ for the variable $U$ can be realized by means of $M$ boundary control functions.

Remark 5.2. Lemmas 1.1 and 1.6 are always available for the reduced coupled system for the variable $W$.

The next question is for any given coupling $N \times N$ matrices $A, B$ and $\bar{B}$, how to guarantee the existence of such $M \times N$ matrix $(\mathrm{H}$ and $M \times M$ matrices $\widetilde{A}, \widetilde{B}$ and $\widetilde{\bar{B}}$ in Theorem 5.1.

We first consider the case $M=N-1$.

Theorem 5.3. Suppose that $M=N-1$. For the coupled system of wave equations (1.2), (1.5) and (1.7), if the coupling matrices $A, B$ and $\bar{B}$ possess a common real right eigenvector $X \in \mathbb{R}^{N} \backslash\{0\}$ :

$$
\begin{aligned}
& A X=\widetilde{\widetilde{a}} X, \\
& B X=\widetilde{\widetilde{b}} X, \\
& \bar{B} X=\widetilde{\widetilde{b}} X,
\end{aligned}
$$

where $\widetilde{\widetilde{a}}, \widetilde{\widetilde{b}}$ and $\widetilde{\bar{b}}$ are corresponding eigenvalues, respectively, then there exist an $(N-1) \times N$ full row-rank matrix H $=\left(\theta_{i j}\right)$ and $(N-1) \times(N-1)$ matrices $\widetilde{A}, \widetilde{B}$ and $\widetilde{\bar{B}}$ such that (5.11)-(5.13) hold. Moreover, $X$ constitutes a system of basic solutions to the linear algebraic system 


$$
\text { (H) } Y=0 .
$$

Proof. There exist $(N-1)$ linearly independent column vectors $Y_{1}, \ldots, Y_{N-1}$ perpendicular to $X$;

$$
\left\langle X, Y_{j}\right\rangle=0, \quad j=1, \ldots, N-1
$$

Thus, noting (5.14) we have

$$
\left\langle X, A^{T} Y_{j}\right\rangle=\left\langle A X, Y_{j}\right\rangle=0, \quad j=1, \ldots, N-1 .
$$

Then,

$$
A^{T} Y_{j}=\sum_{k=1}^{N-1} \widehat{a}_{j k} Y_{k}, \quad j=1, \ldots, N-1,
$$

where $\widehat{a}_{j k}$ are constants. Let

$$
\widetilde{A}=\left(\widehat{a}_{j k}\right)^{T}=\left(\widetilde{a}_{j k}\right)_{(N-1) \times(N-1)} .
$$

It follows from (5.20) that

$$
A^{T}\left(Y_{1}, \ldots, Y_{N-1}\right)=\left(Y_{1}, \ldots, Y_{N-1}\right) \widetilde{A}^{T}
$$

then, taking

$$
\text { H }=\left(Y_{1}, \ldots, Y_{N-1}\right)^{T},
$$

we get (5.11). Moreover, it is easy to see that $X$ constitutes a system of basic solutions to the linear algebraic system (5.17).

The proof of (5.12)-(5.13) is completely similar.

Remark 5.4. In case (1), $M=N-1$, noting (5.6), $X=(1, \ldots, 1)^{T}$ is a system of basic solutions to (5.17). The requirement that the coupling matrices $A, B$ and $\bar{B}$ possess a common right eigenvector $X$ implies (2.2)-(2.4). Thus, Theorem 5.3 leads to the conclusion given in Section 2.

Remark 5.5. In case (2), $M=N-1$, noting $(5.8), X=\overbrace{0, \ldots, 0,0}^{m} \overbrace{1, \ldots, 1}^{N-m})^{T}$ is a system of basic solutions to (5.17). The requirement that the coupled matrices $A, B$ and $\bar{B}$ possess a common right eigenvector $X$ implies (4.3)-(4.6). Thus, the conclusion given in Section 4 follows from Theorem 5.3.

On the other hand, we have

Theorem 5.6. Suppose that $M=N-1$. If the coupled system (1.2), (1.5) and (1.7) possesses the generalized exact boundary synchronization associated with $\mathrm{H}$, but is not exactly boundary null controllable, then the coupling matrices $A, B$ and $\bar{B}$ must have a common real right eigenvector $X$, and $X$ constitutes a system of basic solutions to the linear algebraic system (5.17).

Proof. Since the rank of $\mathrm{H}$ is $(N-1)$, the linear algebraic system (5.17) possesses a basic solution $X=$ $\left(\alpha_{1}, \ldots, \alpha_{N}\right)^{T} \in \mathbb{R}^{N} \backslash\{0\}$. Thus, when $t \geq T$, it follows from (5.4) that the generalized synchronizable state $U(t, x)=\left(u_{1}(t, x), \ldots, u_{N}(t, x)\right)^{T}$ can be written as

$$
u_{i}(t, x)=\alpha_{i} \widetilde{\widetilde{u}}(t, x), \quad 0 \leq x \leq L,
$$

where $\widetilde{\widetilde{u}}=\widetilde{\widetilde{u}}(t, x)$ is a $C^{2}$ function.

Substituting (5.24) into (1.4), when $t \geq T$ we have

$$
\alpha_{i}\left(\frac{\partial^{2} \widetilde{\widetilde{u}}}{\partial t^{2}}-\frac{\partial^{2} \widetilde{\widetilde{u}}}{\partial x^{2}}\right)+\left(\sum_{j=1}^{N} a_{i j} \alpha_{j}\right) \widetilde{\widetilde{u}}=0, \quad i=1, \ldots, N .
$$


Let

$$
\aleph=\left\{i \mid \alpha_{i} \neq 0, i=1, \ldots, N\right\} .
$$

When the number of $\aleph$ is bigger than or equal to 2 , it is easy to see form (5.25) that for any given $i, k \in \aleph$,

$$
\frac{1}{\alpha_{i}}\left(\sum_{j=1}^{N} a_{i j} \alpha_{j}\right) \widetilde{\widetilde{u}}=\frac{1}{\alpha_{k}}\left(\sum_{j=1}^{N} a_{k j} \alpha_{j}\right) \widetilde{\widetilde{u}}
$$

then by the non exact null controllability, we get

$$
\frac{1}{\alpha_{i}}\left(\sum_{j=1}^{N} a_{i j} \alpha_{j}\right)=\frac{1}{\alpha_{k}}\left(\sum_{j=1}^{N} a_{k j} \alpha_{j}\right) \stackrel{\text { def. }}{=} \widetilde{\widetilde{a}}, \quad \forall i, k \in \aleph
$$

namely, for $i \in \aleph$ we have

$$
\sum_{j=1}^{N} a_{i j} \alpha_{j}=\widetilde{\widetilde{a}} \alpha_{i}
$$

where $\widetilde{\widetilde{a}}$ is a constant independent of $i \in \aleph$. On the other hand, for $i \notin \aleph$, since $\alpha_{i}=0$, by (5.25) and the non exact null controllability, (5.27) is still valid.

When the number of $\aleph$ is equal to 1 , without loss of generality, we may suppose that $\aleph=\{1\}$, namely, $\alpha_{1} \neq 0$ and $\alpha_{2}=\ldots \alpha_{N}=0$. Taking $\widetilde{a}=a_{11}$, we still have (5.27) for $i=1$. Moreover, as in the previous situation, (5.27) is still valid for $i \notin \aleph$.

Thus, for $i=1 \ldots, N$ we always have (5.27), where $\widetilde{\widetilde{a}}$ is independent of $i$. This proves (5.14). Moreover, it follows from (5.25) and (5.14) that $\widetilde{\widetilde{u}}=\widetilde{\widetilde{u}}(t, x)$ satisfies the wave equation (2.6) for $t \geq T$.

We next prove (5.15). The proof of (5.16) is similar.

Substituting (5.24) into the boundary condition (1.5c), for $t \geq T$ we have

$$
x=0: \quad \alpha_{i} \frac{\partial \widetilde{\widetilde{u}}}{\partial x}-\left(\sum_{j=1}^{N} b_{i j} \alpha_{j}\right) \widetilde{\widetilde{u}}=0, \quad i=1, \ldots, N .
$$

As in the proof of Lemma 2.2, we may suppose that $\widetilde{\widetilde{u}}(t, 0) \not \equiv 0$ for $t \geq T$, then similarly we can get

$$
\sum_{j=1}^{N} b_{i j} \alpha_{j}=\widetilde{\widetilde{b}} \alpha_{i}, \quad i=1, \ldots, N
$$

where $\widetilde{\widetilde{b}}$ is a constant independent of $i$. This proves (5.15).

Remark 5.7. As $t \geq T$, for the generalized synchronizable state $U(t, x)=\widetilde{\widetilde{u}}(t, x) X$, where $X=\left(\alpha_{1}, \ldots, \alpha_{N}\right)^{T}$ is a basic solution of (5.17), $\widetilde{\widetilde{u}}=\widetilde{\widetilde{u}}(t, x)$ satisfies the wave equation (2.6) and the boundary conditions (2.19) and (2.20). Then, if we know the initial state $(2.21)$ of $\widetilde{\widetilde{u}}=\widetilde{\widetilde{u}}(t, x)$ at the moment $t=T$, we can completely determine the evolution of $\widetilde{\widetilde{u}}=\widetilde{\widetilde{u}}(t, x)$ with respect to $t$.

Moreover, as in Lemma 2.7, the set of all possible initial data $(\varphi, \psi)$ of $\widetilde{\widetilde{u}}=\widetilde{\widetilde{u}}(t, x)$ is the whole space $C^{2}[0, L] \times C^{1}[0, L]$ associated with the conditions of $C^{2}$ compatibility at the points $(t, x)=(T, 0)$ and $(T, L)$, respectively, with boundary conditions (2.19) and (2.20). 
For the case $M=N-2$, we only consider the special situation that

$$
\text { (H) }=\left(\begin{array}{lll}
\mathrm{H}_{1} & & \\
& & \mathrm{H}_{2}
\end{array}\right)_{(N-2) \times N},
$$

where $\mathrm{H}_{1}$ is a full row-rank $\left(N_{1}-1\right) \times N_{1}$ matrix, while $\mathrm{H}_{2}$ is a full row-rank $\left(N_{2}-1\right) \times N_{2}$ matrix with $N_{1}+N_{2}=N$.

Let

$$
A=\left(\begin{array}{ll}
A_{11} & A_{12} \\
A_{21} & A_{22}
\end{array}\right), \quad \widetilde{A}=\left(\begin{array}{cc}
\widetilde{A}_{11} & \widetilde{A}_{12} \\
\widetilde{A}_{21} & \widetilde{A}_{22}
\end{array}\right) \text { etc. },
$$

where $A_{11}$ (resp. $\left.\widetilde{A}_{11}\right)$ is an $N_{1} \times N_{1}$ (resp. $\left.\left(N_{1}-1\right) \times\left(N_{1}-1\right)\right)$ matrix, $A_{21}$ (resp. $\left.\widetilde{A}_{21}\right)$ is an $N_{2} \times N_{1}$ (resp. $\left.\left(N_{2}-1\right) \times\left(N_{1}-1\right)\right)$ matrix, $A_{12}\left(\right.$ resp. $\left.\widetilde{A}_{12}\right)$ is an $N_{1} \times N_{2}\left(\right.$ resp. $\left.\left(N_{1}-1\right) \times\left(N_{2}-1\right)\right)$ matrix, $A_{22}\left(\right.$ resp. $\left.\widetilde{A}_{22}\right)$ is an $N_{2} \times N_{2}$ (resp. $\left(N_{2}-1\right) \times\left(N_{2}-1\right)$ ) matrix etc. Thus, (5.11)-(5.13) in Theorem 5.1 can be equivalently written as

$$
\begin{array}{ll}
\mathrm{H}_{i} A_{i j}=\widetilde{A}_{i j} \mathrm{H}_{j}, & i, j=1,2, \\
\mathrm{H}_{i} B_{i j}=\widetilde{B}_{i j} \mathrm{H}_{j}, & i, j=1,2, \\
\mathrm{H}_{i} B_{i j}=\bar{B}_{i j} \mathrm{H}_{j}, & i, j=1,2 .
\end{array}
$$

Theorem 5.8. Suppose that $M=N-2$. For the coupled system of wave equations (1.2), (1.5) and (1.7), if there exist real vectors $X_{1} \in R^{N_{1}} \backslash\{0\}$ and $X_{2} \in R^{N_{2}} \backslash\{0\}$ and real numbers $\widetilde{\widetilde{a}}_{i j}$, $\widetilde{\widetilde{b}}_{i j}$ and $\widetilde{\overline{\vec{b}}}_{i j}$ such that

$$
\begin{aligned}
& A_{i j} X_{j}=\widetilde{\widetilde{a}}_{i j} X_{i}, \quad i, j=1,2, \\
& B_{i j} X_{j}=\widetilde{\widetilde{b}}_{i j} X_{i}, \quad i, j=1,2, \\
& \bar{B}_{i j} X_{j}=\widetilde{\bar{b}}_{i j} X_{i}, \quad i, j=1,2 \text {, }
\end{aligned}
$$

then we can find a $(N-2) \times N$ full row-rank matrix $\mathrm{H}=\left(\begin{array}{l}\mathrm{H}_{1} \\ \left.{ }^{1} \mathrm{H}_{2}\right)\end{array}\right)$ and $(N-2) \times(N-2)$ matrices $\widetilde{A}, \widetilde{B}$ and $\widetilde{\bar{B}}$ such that (5.31)-(5.33) hold, moreover, $X_{1}$ (resp. $X_{2}$ ) constitutes a system of basic solutions to the linear algebraic system

$$
\text { (H) } \left.Y_{1}=0 \text { (resp. } \mathrm{H}_{2} Y_{2}=0\right) \text {. }
$$

Proof. Specially taking $i=j$ in (5.34)-(5.36), we get

$$
\begin{aligned}
A_{i i} X_{i} & =\widetilde{\widetilde{a}}_{i i} X_{i}, \quad i=1,2, \\
B_{i i} X_{i} & =\widetilde{\widetilde{b}}_{i i} X_{i}, \quad i=1,2, \\
\bar{B}_{i i} X_{i} & =\widetilde{\widetilde{b}}_{i i} X_{i}, \quad i=1,2 .
\end{aligned}
$$

Then, by the proof of Theorem 5.2, there exist $N_{1}-1$ (resp. $N_{2}-1$ ) linearly independent column vectors $Y_{1}, \ldots, Y_{N_{1}-1} \in \mathbb{R}^{N_{1}} \backslash\{0\}$ (resp. $Y_{N_{1}}, \ldots, Y_{N-2} \in \mathbb{R}^{N_{2}} \backslash\{0\}$ ) perpendicular to $X_{1}$ (resp. $X_{2}$ ) such that (5.31)-(5.33) hold for $i=j$ with some matrices $\widetilde{A}_{i i}, \widetilde{B}_{i i}$ and $\widetilde{\bar{B}}_{i i}$ and

$$
\mathrm{H}_{1}=\left(Y_{1}, \ldots, Y_{N_{1}-1}\right)^{T}, \quad \mathrm{H}_{2}=\left(Y_{N_{1}}, \ldots, Y_{N-2}\right)^{T} .
$$


We now prove that there exist matrices $\widetilde{A}_{i j}, \widetilde{B}_{i j}$ and $\widetilde{\bar{B}}_{i j}$ for $i \neq j$ such that (5.31)-(5.33) are still valid for $i \neq j$, in which $\mathrm{H}_{1}$ and $\mathrm{H}_{2}$ are given by (5.41). For fixing the data, in what follows we only prove (5.31) for $i=1$ and $j=2$.

Noting (5.34), we have

$$
\left\langle X_{2}, A_{12}^{T} Y_{j}\right\rangle=\left\langle A_{12} X_{2}, Y_{j}\right\rangle=\left\langle\widetilde{\widetilde{a}}_{12} X_{1}, Y_{j}\right\rangle=0, \quad j=1, \ldots, N_{1}-1 .
$$

Then

$$
A_{12}^{T} Y_{j}=\sum_{k=N_{1}}^{N-2} \widehat{a}_{j k} Y_{k}, \quad j=1, \ldots, N_{1}-1
$$

where $\widehat{a}_{j k}$ are constants. Let

$$
\widetilde{A}_{12}=\left(\widehat{a}_{j k}\right)^{T}=\left(\widetilde{a}_{j k}\right)
$$

By (5.43), we have

$$
A_{12}^{T}\left(Y_{1}, \ldots, Y_{N_{1}-1}\right)=\left(Y_{1}, \ldots, Y_{N_{1}-1}\right) \widetilde{A}_{12}^{T},
$$

which together with (5.41) leads to (5.31) for $i=1$ and $j=2$.

Remark 5.9. In case (3), $M=N-2$, noting $(5.10)$, we have $X_{1}=(\overbrace{1, \ldots, 1}^{m})^{T}$ and $X_{2}=(\overbrace{1, \ldots, 1}^{N-m})^{T}$. The requirements (5.34)-(5.36) imply (3.3)-(3.4) and (3.7)-(3.8), then Theorem 5.8 leads to the conclusion given in Section 3.

By (5.29) and noting that the rank of both $\mathrm{H}_{1}$ and $\mathrm{H}_{2}$ is $(N-1)$, linear algebraic system $(5.37)$ possesses a basic solution $X_{1}=\left(\alpha_{1}, \ldots, \alpha_{N_{1}}\right)^{T} \in \mathbb{R}^{N_{1}} \backslash\{0\}$ (resp. $\left.X_{2}=\left(\alpha_{N_{1}+1}, \ldots, \alpha_{N}\right)^{T} \in \mathbb{R}^{N_{2}} \backslash\{0\}\right)$. Thus, noting (5.4), as $t \geq T$ the generalized synchronizable state $U(t, x)=\left(u_{1}(t, x), \ldots, u_{N}(t, x)\right)^{T}$ can be written as

$$
\begin{aligned}
& u_{p}(t, x)=\alpha_{p} \widetilde{\widetilde{u}}_{1}(t, x), \quad p=1, \ldots, N_{1}, \quad 0 \leq x \leq L, \\
& u_{q}(t, x)=\alpha_{q} \widetilde{\widetilde{u}}_{2}(t, x), \quad q=N_{1}+1, \ldots, N, \quad 0 \leq x \leq L .
\end{aligned}
$$

Similarly to Theorem 5.6, we have

Theorem 5.10. Suppose that $M=N-2$. If the coupled system (1.2), (1.5) and (1.7) possesses the generalized exact boundary synchronization associated with (5.29), and at least for an initial state $(\Phi(x), \Psi(x)), \widetilde{\widetilde{u}}_{1}(t, x)$ and $\widetilde{\widetilde{u}}_{2}(t, x)$ given by (5.46)-(5.47) for the generalized synchronizable state are linearly independent. Then there exist real vectors $X_{1} \in \mathbb{R}^{N_{1}} \backslash\{0\}$ and $X_{2} \in \mathbb{R}^{N_{2}} \backslash\{0\}$ and real numbers $\widetilde{\widetilde{a}}_{i j}(i, j=1,2)$ such that (5.34) holds.

Proof. Substituting (5.46)-(5.47) into (1.4), we have

$$
\begin{aligned}
& \alpha_{i}\left(\frac{\partial^{2} \widetilde{\widetilde{u}}_{1}}{\partial t^{2}}-\frac{\partial^{2} \widetilde{\widetilde{u}}_{1}}{\partial x^{2}}\right)+\left(\sum_{p=1}^{N_{1}} a_{i p} \alpha_{p}\right) \widetilde{\widetilde{u}}_{1}+\left(\sum_{q=N_{1}+1}^{N} a_{i q} \alpha_{q}\right) \widetilde{\widetilde{u}}_{2}=0, \quad i=1, \ldots, N_{1}, \\
& \alpha_{i}\left(\frac{\partial^{2} \widetilde{\widetilde{u}}_{2}}{\partial t^{2}}-\frac{\partial^{2} \widetilde{\widetilde{u}}_{2}}{\partial x^{2}}\right)+\left(\sum_{p=1}^{N_{1}} a_{i p} \alpha_{p}\right) \widetilde{\widetilde{u}}_{1}+\left(\sum_{q=N_{1}+1}^{N} a_{i q} \alpha_{q}\right) \widetilde{\widetilde{u}}_{2}=0, \quad i=N_{1}+1, \ldots, N .
\end{aligned}
$$

Let

$$
\aleph_{1}=\left\{p \mid \alpha_{p} \neq 0, p=1, \ldots, N_{1}\right\}
$$


When the number $\aleph_{1}$ is bigger than or equal to 2 , it is easy to see from (5.48) that for any given $i, k \in \aleph_{1}$

$$
\begin{aligned}
& \frac{1}{\alpha_{i}}\left[\left(\sum_{p=1}^{N_{1}} a_{i p} \alpha_{p}\right) \widetilde{\widetilde{u}}_{1}+\left(\sum_{q=N_{1}+1}^{N} a_{i q} \alpha_{q}\right) \widetilde{\widetilde{u}}_{2}\right] \\
= & \frac{1}{\alpha_{k}}\left[\left(\sum_{p=1}^{N_{1}} a_{k p} \alpha_{p}\right) \widetilde{\widetilde{u}}_{1}+\left(\sum_{q=N_{1}+1}^{N} a_{k q} \alpha_{q}\right) \widetilde{\widetilde{u}}_{2}\right],
\end{aligned}
$$

then, since we may assume that $\widetilde{\widetilde{u}}_{1}$ and $\widetilde{\widetilde{u}}_{2}$ are linearly independent, we get

$$
\begin{aligned}
& \frac{1}{\alpha_{i}}\left(\sum_{p=1}^{N_{1}} a_{i p} \alpha_{p}\right)=\frac{1}{\alpha_{k}}\left(\sum_{p=1}^{N_{1}} a_{k p} \alpha_{p}\right) \stackrel{\text { def. }}{=} \widetilde{\widetilde{a}}_{11}, \quad \forall i, k \in \aleph_{1}, \\
& \frac{1}{\alpha_{i}}\left(\sum_{q=N_{1}+1}^{N} a_{i q} \alpha_{q}\right)=\frac{1}{\alpha_{k}}\left(\sum_{q=N_{1}+1}^{N} a_{k q} \alpha_{q}\right) \stackrel{\text { def. }}{=} \widetilde{\widetilde{a}}_{12}, \quad \forall i, k \in \aleph_{1},
\end{aligned}
$$

namely, for $i \in \aleph_{1}$, we have

$$
\begin{aligned}
\sum_{p=1}^{N_{1}} a_{i p} \alpha_{p} & =\widetilde{\widetilde{a}}_{11} \alpha_{i}, \\
\sum_{q=N_{1}+1}^{N} a_{i q} \alpha_{q} & =\widetilde{\widetilde{a}}_{12} \alpha_{i},
\end{aligned}
$$

where $\widetilde{\widetilde{a}}_{11}$ and $\widetilde{\widetilde{a}}_{12}$ are constants in dependent of $i \in \aleph_{1}$. On the other hand, for $i \notin \aleph_{1}$, since $\alpha_{i}=0$, by $(5.48)$ and noting that $\widetilde{\widetilde{u}}_{1}$ and $\widetilde{\widetilde{u}}_{2}$ are linearly independent, (5.51)-(5.52) are still valid.

When the number of $\aleph_{1}$ is equal to 1 , without loss of generality, we may suppose that $\aleph_{1}=\{1\}$, namely, $\alpha_{1} \neq 0$ and $\alpha_{2}=\ldots=\alpha_{N_{1}}=0$. Taking $\widetilde{\widetilde{a}}_{11}=a_{11}$ and $\widetilde{\widetilde{a}}_{12}=\frac{1}{\alpha_{1}}\left(\sum_{q=N_{1}+1}^{N} a_{1 q} \alpha_{q}\right)$, we still have (5.51)-(5.52) for $i=1$. Moreover, as in the previous situation, (5.51)-(5.52) are still valid for $i \notin \aleph_{1}$.

Thus, for $i=1, \ldots, N$, we always have (5.51)-(5.52), where $\widetilde{\widetilde{a}}_{11}$ and $\widetilde{\widetilde{a}}_{12}$ are independent of $i$. This proves (5.34) for $i=j=1$ and $i=1, j=2$.

Similarly, by (5.49), we get (5.34) for $i=2, j=1$ and $i=j=2$.

Remark 5.11. As $t \geq T$, for the generalized sychronizable state $U(t, x)=\left(\begin{array}{c}\tilde{\tilde{u}}_{1}(t, x) X_{1} \\ \tilde{\tilde{u}}_{2}(t, x) X_{2}\end{array}\right)$ given by $(5.46)-(5.47)$, where $X_{1}=\left(\alpha_{1}, \ldots, \alpha_{N_{1}}\right)^{T}$ (resp. $\left.X_{2}=\left(\alpha_{N_{1}+1}, \ldots, \alpha_{N}\right)^{T}\right)$ is a basic solution to $(5.37), \tilde{\widetilde{U}}(t, x)=\left(\begin{array}{c}\tilde{\tilde{u}}_{1}(t, x) \\ \tilde{u}_{2}(t, x)\end{array}\right)$ satisfies the coupled system (3.16) of wave equations with (3.17), in which $\underset{\widetilde{\widetilde{a}}}{\widetilde{\widetilde{b}}}(i, j=1,2)$ are given by $(5.34)$, and the boundary conditions (3.18) and (3.19) with (3.20), in which $\widetilde{\widetilde{b}}_{i j}$ and $\widetilde{\widetilde{b}}_{i j}(i, j=1,2)$ are given by (5.35)(5.36). Hence, if we know the initial state $(3.21)$ of $\widetilde{\widetilde{U}}=\widetilde{\widetilde{U}}(t, x)$ at the moment $t=T$, then the evolution of $\widetilde{\widetilde{U}}=\widetilde{\widetilde{U}}(t, x)$ with respect to $t$ can be completely determined.

Moreover, any given state $\left(\varphi^{(1)}(x), \varphi^{(2)}(x)\right)^{T}$ and $\left(\psi^{(1)}(x), \psi^{(2)}(x)\right)^{T}$ in $\left(C^{2}[0, L]\right)^{2} \times\left(C^{1}[0, L]\right)^{2}$, satisfying the conditions of $C^{2}$ compatibility at the points $(t, x)=(T, 0)$ and $(T, L)$, respectively, with boundary conditions (3.18) and (3.19), belongs to the attainable set of the initial data of $\widetilde{\widetilde{U}}=\widetilde{\widetilde{U}}(t, x)$.

Thus, at least for some initial states $(\Phi(x), \Psi(x)), \widetilde{\widetilde{u}}_{1}(t, x)$ and $\widetilde{\widetilde{u}}_{2}(t, x)$ are linearly independent on $t=T$ then for $t \geq T$. This shows that the corresponding requirement given in Theorem 5.5 is reasonable. 
Remark 5.12. The situation $M=N-k(k>2)$ can be similarly discussed.

\section{REMARKS}

Remark 6.1. The whole previous discussion is still valid for the case of two-sided controls, provided that (2.8) is replaced by

$$
T>L
$$

and there is an inhomogeneous term $\bar{H}(t)$ on the right-hand side of (1.7).

Remark 6.2. It is possible that, different from Lemma 1.6, in the case of one-sided controls, the exact boundary (null) controllability may be realized (perhaps in a weaker sense) by means of $M(<N)$ boundary controls for some special coupled systems of $N$ wave equations $(c f$. [1-3], [12]). Thus, the exact boundary synchronization (perhaps in a weaker sense) could be realized by only $P(<M)$ boundary controls. In a forthcoming paper we will discuss this situation in detail.

Remark 6.3. The problem of synchronization might be also considered as an optimal control problem, where an objective function of the type

$$
\sum_{i=1}^{N-1}\left\|u_{i}-u_{i+1}\right\|^{2}
$$

is minimized. This kind of optimal control is similar to the optimal control of partial differential equations on networks ( $c f$. [5] for an application in flood management) and should be studied in the framework of weak solutions.

\section{REFERENCES}

[1] F. Alabau-Boussouira, Indirect boundary stabilization of weakly coupled hyperbolic systems. SIAM J. Control Optim. 41 (2002) 511-541.

[2] F. Alabau-Boussouira, A two-level energy method for indirect boundary observability and controllability of weakly coupled hyperbolic systems. SIAM J. Control Optim. 42 (2003) 871-906.

[3] F. Alabau-Boussouira, M. Léautaud, Indirect stabilization of locally coupled wave-type systems. ESAIM: COCV 18 (2012) $548-582$.

[4] H. Fujisaka and T. Yamada, Stability theory of synchronized motion in coupled-oscillator systems. Progress Theoret. Phys. 69 (1983) 32-47.

[5] M. Gugat, Optimal boundary control in flood management, Control of Coupled Partial Differential Equations, edited by K. Kunisch, J. Sprekels, G. Leugering and F. Tröltzsch, vol. 155 of Int. Ser. Numer. Math., Birkhäuser Verlag, Basel/Switzerland (2007) 69-94.

[6] Long Hu, Fanqiong Ji and Ke Wang, Exact boundary controllability and exact boundary observability for a coupled system of quasilinear wave equations. Chin. Ann. Math. B 34 (2013) 479-490.

[7] Ch. Huygens, Euvres Complètes, vol. 15, edited by S. and B.V. Zeitlinger, Amsterdam (1967).

[8] Tatsien Li and Yi Jin, Semi-global $C^{1}$ solution to the mixed initial-boundary value problem for quasilinear hyperbolic systems. Chin. Ann. Math. B 22 (2001) 325-336.

[9] Tatsien Li, Exact boundary observability for 1-D quasilinear wave equations. Math. Meth. Appl. Sci. 29 (2006) $1543-1553$.

[10] Tatsien Li, Controllability and Observability for Quasilinear Hyperbolic Systems, vol. 3 of AIMS Ser. Appl. Math. AIMS and Higher Education Press (2010).

[11] Tatsien Li and Bopeng Rao, Strong (weak) exact controllability and strong (weak) exact observability for quasilinear hyperbolic systems. Chin. Annal. Math. B 31 (2010) 723-742.

[12] Tatsien Li and Bopeng Rao, Asymptotic controllability for linear hyperbolic systems. Asymp. Anal. 72 (2011) $169-187$.

[13] Tatsien Li and Bopeng Rao, Exact synchronization for a coupled system of wave equations with Dirichlet boundary controls. Chin. Annal. Math. B 34 (2013) 139-160.

[14] J.-L. Lions, Contrôlabilité Exacte, Perturbations et Stabilization de Systèmes Distribués, Vol. 1, Masson (1988).

[15] J.-L. Lions, Exact controllability, stabilization and perturbations for distributed systems. SIAM Review 30 (1988) 1-68. 
[16] D.L. Russell, Controllability and stabilization theory for linear partial differential equations: Recent progress and open questions. SIAM Review 20 (1978) 639-739.

[17] S. Strogatz, SYNC: The Emerging Science of Spontaneous Order, THEIA, New York (2003).

[18] Ke Wang, Exact boundary controllability for a kind of second-order quasilinear hyperbolic systems. Chin. Ann. Math. B 32 (2011) 803-822.

[19] Chai Wah Wu, Synchronization in Complex Networks of Nonlinear Dynamical Systems. World Scientific (2007).

[20] Lixin Yu, Exact boundary controllability for a kind of second-order quasilinear hyperbolic systems and its applications. Math. Meth. Appl. Sci. 33 (2010) 273-286. 